\title{
Laser Rate Equation Based Filtering for Carrier Recovery in Characterization and Communication
}

Piels, Molly; Iglesias Olmedo, Miguel; Xue, Weiqi; Pang, Xiaodan; Schaffer, Christian; Schatz, Richard; Jacobsen, Gunnar; Tafur Monroy, Idelfonso ; Mørk, Jesper; Popov, Sergei

Total number of authors:

11

Published in:

Journal of Lightwave Technology

Link to article, DOI:

10.1109/JLT.2015.2430373

Publication date:

2015

Link back to DTU Orbit

Citation (APA):

Piels, M., Iglesias Olmedo, M., Xue, W., Pang, X., Schaffer, C., Schatz, R., Jacobsen, G., Tafur Monroy, I., Mørk, J., Popov, S., \& Zibar, D. (2015). Laser Rate Equation Based Filtering for Carrier Recovery in Characterization and Communication. Journal of Lightwave Technology, 33(15), 3271-3279.

https://doi.org/10.1109/JLT.2015.2430373

\section{General rights}

Copyright and moral rights for the publications made accessible in the public portal are retained by the authors and/or other copyright owners and it is a condition of accessing publications that users recognise and abide by the legal requirements associated with these rights.

- Users may download and print one copy of any publication from the public portal for the purpose of private study or research.

- You may not further distribute the material or use it for any profit-making activity or commercial gain

- You may freely distribute the URL identifying the publication in the public portal 


\title{
Laser Rate Equation Based Filtering for Carrier Recovery in Characterization and Communication
}

\author{
Molly Piels, Member, IEEE, Miguel Iglesias Olmedo, Student Member, IEEE, Weiqi Xue, \\ Xiaodan Pang, Member, IEEE, Christian Schäffer, Member, IEEE, Richard Schatz, Gunnar Jacobsen, \\ Idelfonso Tafur Monroy, Senior Member, IEEE, Jesper Mørk, Member, IEEE, Sergei Popov, \\ and Darko Zibar, Member, IEEE
}

\begin{abstract}
We formulate a semiconductor laser rate equationbased approach to carrier recovery in a Bayesian filtering framework. Filter stability and the effect of model inaccuracies (unknown or un-useable rate equation coefficients) are discussed. Two potential application areas are explored: laser characterization and carrier recovery in coherent communication. Two rate equation based Bayesian filters, the particle filter and extended Kalman filter, are used in conjunction with a coherent receiver to measure frequency noise spectrum of a photonic crystal cavity laser with less than $20 \mathrm{nW}$ of fiber-coupled output power. The extended Kalman filter is also used to recover a 28 GBd DP16 QAM signal where a decision-directed phase-locked loop fails.
\end{abstract}

Index Terms-Phase estimation, phase noise, diode lasers, semiconductor lasers, coherent communication, optical communication, quadrature amplitude modulation.

\section{INTRODUCTION}

$\mathbf{S}$ EMICONDUCTOR laser phase noise has been the subject of study in device design [1], [2] and coherent optical communications [3], [4] communities for quite some time. From the laser physics standpoint, measuring the frequency noise (FM) spectrum provides useful information regarding internal laser dynamics. In coherent communication, phase noise is an impairment that needs to be compensated. Historically, these two tasks, characterizing and compensating, have been accomplished using different experimental setups and data analysis. Increasingly, the same digital coherent receivers that are typically used in coherent communication systems are becoming popular tools for characterizing the phase noise of lasers as well [5]-[7], due to the flexibility offered by full-field detection and their increasing ubiquity in laboratories. This

M. Piels, W. Xue, I. Tafur Monroy, J. Mørk, and D. Zibar are with DTU Fotonik, Department of Photonics Engineering, Technical University of Denmark, Kgs. Lyngby 2800, Denmark, e-mail: (mopi@ fotonik.dtu.dk).

M. I. Olmedo, R. Schatz, G. Jacobsen, and S. Popov are with Optics division, Royal Institute of Technology (KTH), Electrum 229, Kista, SE164 40, Sweden, e-mail: (miguelio@kth.se). X. Pang and G. Jacobsen are with Networking and Transmission Laboratory, Acreo AB, SE- 16425 Kista, Sweden.

C. Schäffer is with the Faculty of Electrical Engineering, Helmut Schmidt University, Holstenhofweg 85, 22043 Hamburg, Germany.

This work was supported by the Villum Foundation Young Investigator Program and NATEC Centre of Excellence. Research leading to these results has received funding from the Danish Council for Independent Research, project CORESON. This work was also supported by EU project GRIFFON, gr. no 324391 and project ICONE, gr. no. 608099 .

Copyright (c) 2015 IEEE. Personal use of this material is permitted. However, permission to use this material for any other purposes must be obtained from the IEEE by sending a request to pubs-permissions@iee.org. collapses these two tasks into one, the results of which will generally be improved by a digital filter that more accurately tracks the phase.

It is well-established that optimum tracking is made possible only by taking the probability density functions (PDFs) of all relevant noise sources into account. Most carrier recovery algorithms developed to date have been for lasers with a Lorentzian lineshape [4], [8], which is the result of a Wiener process. However, semiconductor lasers (SCLs) almost never have Lorentzian lineshapes due to relaxation oscillations. Thus we would expect that taking the unique SCL line structure into account when designing a digital filter for carrier phase recovery (CPR) would yield some improvement in performance. Here, we reformulate the SCL rate equations so that they can be used in a Bayesian filtering context [9], [10]. Bayesian filtering, in this case, provides a framework for recursive estimation of the noise variance (FM noise magnitude), while the SCL rate equations specify the shape of that spectrum. We focus on two different Bayesian tracking algorithms: the particle filter (PF) and the extended Kalman filter (EKF). We then apply these filters in two different experiments where other carrier recovery methods were not successful: a measurement of the FM noise spectrum of a photonic crystal $(\mathrm{PhC})$ cavity laser with extremely low output power [11], [12] and demodulation of a $28 \mathrm{GBd}$ DP-16 QAM signal from a semiconductor laser-based transmitter [13], [14].

The remainder of the paper is organized as follows: Section II reformulates the SCL rate equations for use in the Bayesian filtering framework. Section II also discusses some of the practical concerns, inherent limitations, and benefits of this approach. Section III contains further implementation details for the Bayesian filters and reference methods. In Section IV-A, the rate equation based particle filter and extended Kalman filter are applied to the phase noise characterization problem, first for lasers with known FM noise spectra to validate the method, then to a PhC laser with an unknown FM response. We show a significant improvement in sensitivity over a reference method in both cases, which for the $\mathrm{PhC}$ laser made the relevant spectral features visible. Section IV-B discusses the use of the SCL EKF in a 28 GBd DP-16 QAM communication system where the transmit laser has one of several FM noise profiles. We show that this filter can successfully recover the signal where comparable methods based on Lorentzian laser phase noise models fail. 


\section{SEMICONDUCTOR LASER PHASE TRACKING IN A BAYESIAN FRAMEWORK}

A Bayesian filter is a set of recursion equations that use a system model and measurements to estimate the probability density function (PDF) associated with an underlying process. For carrier phase recovery, the underlying process is the phase evolution. The associated PDF describes the noise that causes phase fluctuations. Bayesian filters are typically formulated for systems described in the state space by a pair of equations of the form

$$
\begin{aligned}
& \mathbf{x}_{\mathbf{n}}=f\left(\mathbf{x}_{\mathbf{n}-\mathbf{1}}, \mathbf{w}_{\mathbf{n}-\mathbf{1}}\right) \\
& \mathbf{y}_{\mathbf{n}}=g\left(\mathbf{x}_{\mathbf{n}}, \mathbf{v}_{\mathbf{n}}\right)
\end{aligned}
$$

where $\mathbf{x}$ is a vector describing the internal state of the process, $\mathbf{y}$ is the measurement output, $f$ and $g$ are functions that describe the process evolution and measurement, respectively, and $\mathbf{w}$ and $\mathbf{v}$ are noise terms. For carrier phase recovery, $\mathbf{x}$ describes the current phase of the laser and $f$ describes how this is expected to change over the course of one sampling period. The measurement vector $\mathbf{y}$ consists of the electrical outputs of a coherent receiver, and is degraded by receiver and channel noise $\mathbf{v}$. When both $f$ and $g$ in (1) are linear and both $\mathbf{w}$ and $\mathbf{v}$ are Gaussian, the Kalman filter equations provide an optimal solution to the Bayesian tracking problem. For laser phase tracking using a coherent receiver, $f$ is linear and $\mathbf{w}$ and $\mathbf{v}$ are Gaussian, but $g$ is a nonlinear function. As a result, the Kalman filter equations are not optimal, and an alternative approach is necessary. For optical communication applications, we use the extended Kalman filter (EKF), which linearizes the measurement equation around the current value of the state vector [15]. It is generally more accurate to use filtering recursion equations specifically formulated for the nonlinear case, such as the particle filter (PF) [10], [16], which we also apply to the laser characterization problem, or the unscented Kalman filter (UKF) [17], [18], though these techniques have increased computational complexity.

\section{A. Process model}

The phase tracking algorithm can account for semiconductor laser dynamics by using appropriate expressions for $f$ and $\mathbf{x}$ in (1). The state vector $\mathbf{x}$ is usually defined to include the phase difference $\phi$ and the frequency offset $\Omega$ between the transmitter and local oscillator. By adding terms to $\mathbf{x}$, relaxation oscillations can also be included in the process equation.

For a laser with a Lorentzian lineshape, the process model given by $f$ and $\mathbf{w}$ describes a random walk:

$$
\mathbf{x}_{\mathbf{n}} \triangleq\left[\begin{array}{l}
\Omega_{n} \\
\phi_{n}
\end{array}\right]=\left[\begin{array}{c}
\Omega_{n-1} \\
\phi_{n-1}
\end{array}\right]+\mathbf{w}_{\mathbf{n}-\mathbf{1}}
$$

where $\mathbf{w}_{\mathbf{n}-\mathbf{1}}=[0 ; \mathcal{N}(0,2 \pi \tau \Delta \nu)]$ is a vector of Gaussian noise sources with zero mean and a variance proportional to the Lorentzian linewidth of the laser $\Delta \nu$ and the sampling period $\tau$. For a semiconductor laser, the phase is usually modeled using the continuous-time semiconductor laser rate equations [2]:

$$
\begin{aligned}
\frac{d \phi}{d t} & =\alpha G_{N} N+F_{\phi} \\
\frac{d \rho}{d t} & =-\Gamma_{I} \rho+G_{N} I_{0} N+F_{\rho} \\
\frac{d N}{d t} & =-G \rho-\Gamma_{N} N+F_{N}
\end{aligned}
$$

where $\phi$ is the phase, $\rho$ is the output intensity perturbation, and $N$ is the excess carrier concentration. The $F_{x}$ terms are the Langevin noise terms associated with each of these variables and are zero-mean Gaussian-distributed noise sources. The variables $\phi, \rho$, and $N$ are used as the state variables in the discrete state-space system model after normalization. Normalization is necessary (when a physically meaningful system model is used) because $\phi, \rho$, and $N$ have dramatically different magnitudes; we normalized each variable to the expected variance of the associated Langevin term. The remaining parameters $\alpha, G_{N}, \Gamma_{I}, \Gamma_{N}, I_{0}$, and $G$ are the linewidth enhancement factor, the differential gain, intensity damping factor, carrier damping factor, steady-state output field intensity, and gain, respectively. These parameters describe laser dynamics and it is often possible to estimate them from steady-state data (e.g. light-intensity-voltage curves). Using the Euler-Maruyama method [19], this system of equations can be rewritten in discrete form:

$$
\left[\begin{array}{c}
\Omega_{n} \\
\phi_{n} \\
\rho_{n} \\
N_{n}
\end{array}\right]=\left(\mathbf{I}+\tau\left[\begin{array}{cccc}
0 & 0 & 0 & 0 \\
1 / \tau & 0 & 0 & \alpha G_{N} \\
0 & 0 & -\Gamma_{I} & G_{N} I_{0} \\
0 & 0 & -G & -\Gamma_{N}
\end{array}\right]\right)\left[\begin{array}{c}
\Omega_{n-1} \\
\phi_{n-1} \\
\rho_{n-1} \\
N_{n-1}
\end{array}\right]+\left[\begin{array}{c}
F_{\Omega, n} \\
F_{\phi, n} \\
F_{\rho, n} \\
F_{N, n}
\end{array}\right]
$$

where $\tau$ is the sampling period (typically the symbol period, for communication systems) and $I$ is the $4 \times 4$ identity matrix. Here, we have added the normalized frequency $\Omega$ to include flicker noise [15], [20]. This can be written more compactly as

$$
\mathbf{x}_{\mathbf{n}}=\mathbf{A} \mathbf{x}_{\mathbf{n}-\mathbf{1}}+\mathbf{w}_{\mathbf{n}-\mathbf{1}}
$$

A typical output frequency noise spectrum is shown in Fig. 1. This spectrum can be completely described by five parameters: $\alpha, f_{R}, K, \Delta \nu$, and $f_{\text {cross }}$. The linewidth enhancement factor $\alpha$ is the same as defined in (3), while $f_{R}$ and $K$ are related to (3) by

$$
\left(2 \pi f_{R}\right)^{2}=\Gamma_{I} \Gamma_{N}+G_{N} G I_{0}
$$

and

$$
K f_{R}^{2}=\Gamma_{I}+\Gamma_{N}
$$

The Lorentzian linewidth $\Delta \nu$ and the $1 / f$ noise crossing frequency $f_{\text {cross }}$ are given by the magnitudes of the variances of the noise terms and do not depend on the coefficients of A.

In the process model described above, we have neglected the contribution of the local oscillator to the phase. For characterization applications, this is a reasonable assumption because the device under test typically has a linewidth much larger than the linewidth of the LO. For communication systems using semiconductor lasers, we also focus on the case where transmitter phase noise is dominant. This is partially because it becomes difficult to separate the effect of equalization 


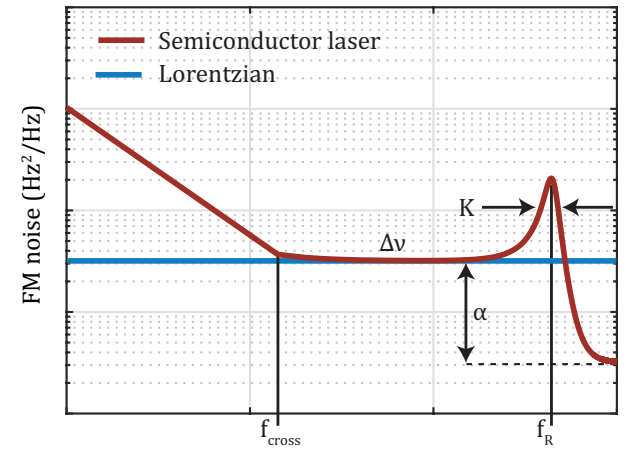

Fig. 1. Power spectral density of frequency noise given by the rate equation (SCL) model and Lorentzian model. Definitions of the parameters that describe this spectrum, $\alpha, f_{R}, K, \Delta \nu$, and $f_{\text {cross }}$, are given in the figure.

enhanced phase noise [21] from CPR algorithm performance when local oscillator phase noise is included. Extending this technique to the case of semiconductor lasers as both $\mathrm{LO}$ and transmitter involves, at most, adding one more pair of terms ( $\rho$ and $N$ ) to (4) to account for an additional relaxation oscillation peak.

\section{B. Measurement model}

The measurement $\mathbf{y}$ is composed of the in-phase and quadrature components of the signal. In this case, the measurement equation (1) becomes

$$
\mathbf{y}_{\mathbf{n}}=\left[\begin{array}{l}
y_{I, n} \\
y_{Q, n}
\end{array}\right]=C\left[\begin{array}{c}
\cos \left(\phi_{n}+\Omega_{n} n\right) \\
\sin \left(\phi_{n}+\Omega_{n} n\right)
\end{array}\right]+\mathbf{v}_{\mathbf{n}}
$$

where $C$ is a scalar (the amplitude) and $\mathbf{v}$ is a $2 \times 1$ (typically Gaussian) measurement noise vector. Within phase tracking applications, the signal is normalized and the effect of modulation is removed so that $C=1$. At sufficiently high signal to noise ratio (and assuming $C=1$ ), it can be shown [3] that applying the inverse of the nonlinear function

$$
y_{n}^{\prime}=\operatorname{unwrap}\left(\arctan \left(\frac{y_{Q, n}}{y_{I, n}}\right)\right)=\phi_{n}+\Omega_{n} n+v_{n}^{\prime}
$$

yields a linear function of $\phi$ and $\Omega$ with Gaussian-distributed noise $v^{\prime}$. Though here we focus on Bayesian filtering approaches formulated to deal with a nonlinear measurement equation, (9) means that the relatively computationally efficient Kalman filter may provide good performance in applications where high SNR is expected.

\section{Filter stability}

The extended Kalman filter and particle filter can be considered infinite impulse response implementations of a Wiener filter for carrier recovery. Relative to finite impulse response approaches, this results in reduced computational complexity, but also increased susceptibility to numerical errors [22], and potential filter instability. For systems like semiconductor lasers with unobserved variables, the filter stability condition is the same as the discrete system stability condition [23]. In terms of the state transition matrix $\mathbf{A}$, this can be expressed as

$$
\forall i,\left|1+\lambda_{i}\right| \leq 1
$$

where $\lambda_{i}$ is one of four eigenvalues of $\mathbf{A}$. For the expression in (4) two of these eigenvalues (the ones associated with the flicker and Lorentzian noise) are unity, and the other two (typically complex) eigenvalues can be expressed in terms of $f_{R}$ and $K$ to form a more specific criterion:

$$
\left|1-\frac{\tau K f_{R}^{2}}{2}+\tau f_{R} \sqrt{\left(\frac{K f_{R}}{2}\right)^{2}-(2 \pi)^{2}}\right| \leq 1 .
$$

In general, decreasing $K$ (which will raise and narrow the peak in Fig. 1) and increasing the resonance frequency $f_{R}$ will decrease the chance that a matched filter is stable at a particular sampling rate. Increasing the sampling rate always has the effect of improving filter stability.

\section{Process model approximation}

It is typically necessary to use a state transition matrix for filtering that is different from the state transition matrix that best describes the system. For device characterization at low SNR, this is because not all values in (3) are known before the data are processed. For coherent communication, the relevant laser parameters may be known in advance, but the corresponding state transition matrix may not yield a stable filter. As a general principle, decreasing $K$ will both incur a larger phase noise penalty for a given Lorentzian linewidth [14] and increase the left hand side of (11). Thus the more detrimental the non-Lorentzian lineshape of the laser is to system performance, the more likely it is to be necessary to specify $\mathbf{A}$ in such a way that the system model is not completely consistent with the expected FM noise spectrum.

The effect of mismatch between the state transition matrix used for filtering and a more accurate system description is best quantified in the frequency domain. Minimizing the mean-squared error (MSE) estimate of the phase by tuning the coefficients of $\mathbf{A}$ is equivalent (for this system model) to minimizing [24]

$$
V=\int_{-f_{s} / 2}^{f_{s} / 2}|\hat{G}(f)-G(f)|^{2} d f
$$

where $f_{s}$ is the sampling frequency, $G(f)$ is the Fourier transform of the system impulse response, $G$ corresponds to the true system, and $\hat{G}$ corresponds to the approximation. For this system model, $V$ will be directly proportional to the MSE with a proportionality constant that depends on the sampling rate and Lorentzian linewidth of the laser. The Fourier transform of $G$ can be approximated as $\sqrt{S_{\phi \phi}(f)}$, where $S_{\phi \phi}(f)$ is the power spectral density of the phase noise (not FM noise). In terms of $\alpha, f_{R}$, and $K$,

$$
S_{\phi \phi}(f)=\left(\frac{1}{2 \pi f}\right)^{2} \frac{\Delta \nu}{\pi}\left[1+\frac{\alpha^{2} f_{R}^{4}}{\left(f_{R}^{2}-f^{2}\right)^{2}+\left(\frac{K}{2 \pi} f_{R}^{2}\right)^{2} f^{2}}\right]
$$

Fig. 2 and Fig. 3 show contour plots of $\log _{10}(V)$ as a function of the estimated values used for $\hat{f}_{R}$ and $\hat{K}$ for FM noise spectra similar to those discussed in Section IV. Together, these two figures provide two specific, though somewhat 


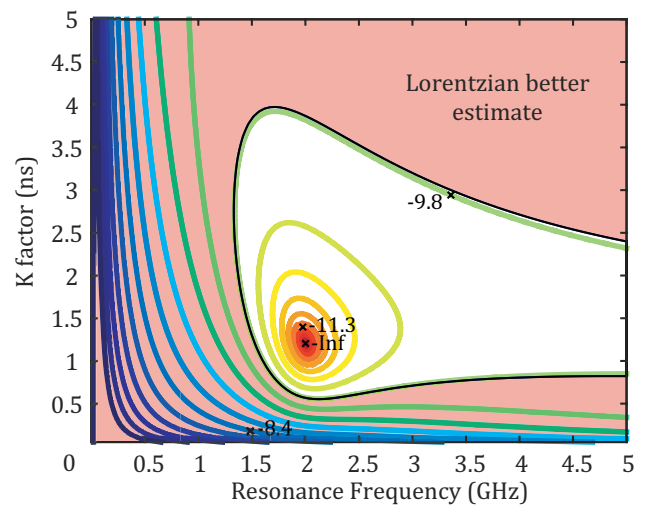

Fig. 2. Contours of equal $\log _{10}(V)$ (defined in (12), $V$ is proportional to the MSE) as a function of model mismatch (error in estimated $\hat{f_{R}}$ and $\hat{K}$ ) for a PhC-like laser phase noise profile $\left(f_{R}=2 \mathrm{GHz}\right.$ and $\left.K=1.2 \mathrm{~ns}\right)$. Areas where a Lorentzian model is expected to yield lower error are shaded red in the figure.

arbitrary, examples that span the full range of likely MSEmodel error relationships; individually, they motivate the different approximation approaches taken in the two experiments. Unsurprisingly, in all cases, using a more accurate model will ensure a lower MSE. The area where the Lorentzian model is expected to perform better than the semiconductor laser model is shaded in red in both figures. The most important effect changing values of $f_{R}$ and $K$ has on these plots is to increase or decrease the size of the region where the semiconductor laser model is expected to perform best. The values of $f_{R}$ and $K$ also affect the relative importance of accurately estimating $\hat{f_{R}}$ and $\hat{K}$.

In Fig. 2, the correct values of $f_{R}$ and $K$ were chosen to be $2 \mathrm{GHz}$ and $1.2 \mathrm{~ns}$, respectively, which produces an FM noise spectrum similar to the photonic crystal cavity laser discussed in Section IV-A. For many reasonable model estimates (e.g. $\hat{f_{R}}=1 \mathrm{GHz}$ and any $\hat{K}$ ), the Lorentzian model is expected to perform several orders of magnitude better than the semiconductor laser model. This indicates that if the laser parameters are not known, the best approach is to start with a Lorentzian model and then use the result to fine-tune $\mathbf{A}$. In Fig. 3, $f_{R}=1 \mathrm{GHz}$ and $K=0.1 \mathrm{~ns}$, which produces an FM noise spectrum likely to cause a large transmission penalty. The figure also shows the region of stability of the filter at $28 \mathrm{GBd}$ (note the laser specified lies outside this region). Since the minimum MSE in the region of stability is lower than the MSE associated with the Lorentzian model, a performance improvement is expected. The figure also implies that the stable filter that provides minimum MSE will use a nearly correct value for $f_{R}$ and an increased value for $K$.

Fig. 4 compares the effect of model error over a broader range of laser parameters: $\log _{10}(V)$ for a Lorentzian model and the best stable SCL model at $28 \mathrm{GBd}$ is plotted over a range of true values of $K$ for several relaxation resonance frequencies. For the Lorentzian model (dashed lines), there is a slight decrease in error as $K$ increases because the phase noise spectrum more closely comes to resemble a Lorentzian. Increasing the resonance frequency also decreases expected MSE because of the $\frac{1}{2 \pi f}$ term in (13). For the SCL model

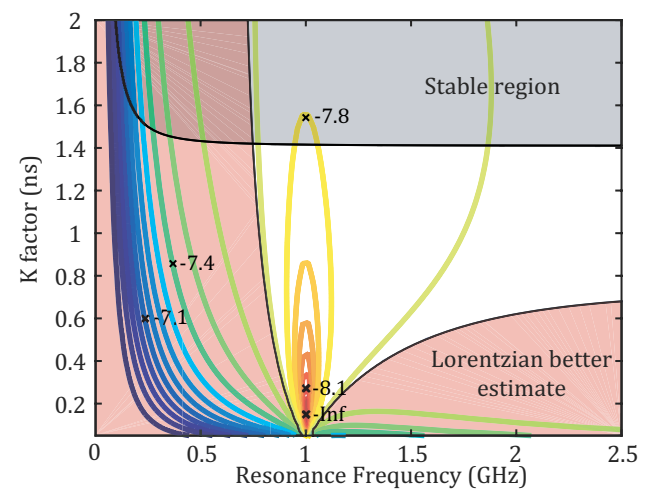

Fig. 3. Contours of equal $\log _{10}(V)$ as a function of model mismatch for a laser phase noise profile that would induce high penalty in a coherent communication system $\left(f_{R}=1 \mathrm{GHz}\right.$ and $\left.K=0.1 \mathrm{~ns}\right)$. The region where the filter would be stable at $28 \mathrm{GBd}$ is shaded in gray. The stability criteria used here is obtained by replacing the 1 in (11) with 0.999 ; this was the criterion used in the experiments.

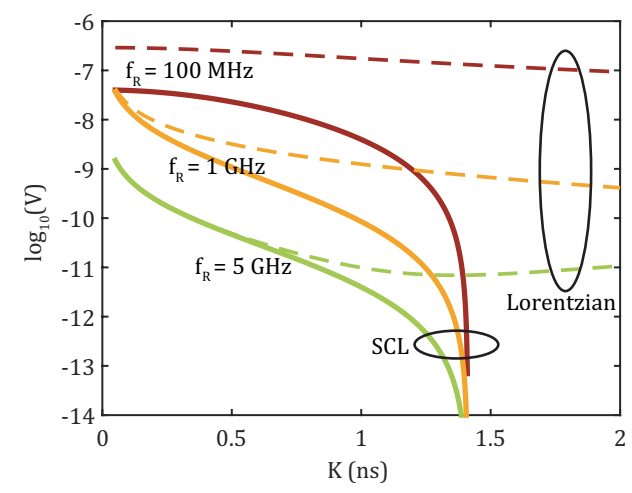

Fig. 4. Expected $V$ (defined in (12), $V$ is proportional to the MSE) for the best stable SCL model at $28 \mathrm{GBd}$ (solid) and Lorentzian model (dashed) as a function of transmitter laser characteristics.

(solid lines), the error goes to zero when $K$ is large enough to permit an accurate stable filter (i.e. $\hat{K}=K$, or $K>1.4$ ns at $28 \mathrm{GBd}$ ), and otherwise follows the same general trends as the Lorentzian model. The effect of increasing the symbol rate is to shift this zero point to a lower value of $K$. The advantage of using the SCL model over the Lorentzian model, indicated by the space between the dashed and solid curves, has a large dependence on the resonance frequency. In particular, the advantage offered by the SCL model is far greater when $f_{R}$ is low. This is also the regime of operation where the Lorentzian model has its worst performance, or where a CPR algorithm based on a Lorentzian model is most likely to fail.

\section{AlgORITHM DESCRIPTION}

\section{A. Extended Kalman Filter}

The extended Kalman filter represents the system state as a Gaussian-distributed variable with mean $\mathrm{x}$ and process noise ( $\mathbf{w}$ in (1)) covariance $\mathbf{P}$. At each time step, first a prior estimate of the current state is generated

$$
\begin{aligned}
p\left(\mathbf{x}_{\mathbf{n}} \mid \mathbf{x}_{\mathbf{n}-\mathbf{1}}\right) & =\mathcal{N}\left(\hat{\mathbf{x}}_{\mathbf{n}}^{-}, \mathbf{P}_{\mathbf{n}}^{-}\right) \\
\hat{\mathbf{x}}_{\mathbf{n}}^{-} & =\mathbf{A} \hat{\mathbf{x}}_{\mathbf{n}-\mathbf{1}}^{-} \\
\mathbf{P}_{\mathbf{n}}^{-} & =\mathbf{A} \mathbf{P}_{\mathbf{n}-\mathbf{1}}^{-} \mathbf{A}^{\mathbf{T}}+\mathbf{Q}
\end{aligned}
$$


where $p()$ denotes a probability, $\mathcal{N}(\mu, \boldsymbol{\Sigma})$ denotes a normal distribution with mean $\mu$ and covariance $\boldsymbol{\Sigma}$, and $\mathbf{Q}$ is the initial guess for $\mathbf{P}$. The associated prior estimate of the measurement is

$$
\begin{aligned}
p\left(\mathbf{y}_{\mathbf{n}} \mid \mathbf{x}_{\mathbf{n}}\right) & =\mathcal{N}\left(\hat{\mathbf{y}}_{\mathbf{n}}^{-}, \mathbf{R}\right) \\
\hat{\mathbf{y}}_{\mathbf{n}}^{-} & =g\left(\hat{\mathbf{x}}_{\mathbf{n}}^{-}\right)
\end{aligned}
$$

where $\mathbf{R}$ is the covariance of the measurement noise ( $\mathbf{v}$ in (1)) and $g$ is the measurement function. Then the Kalman gain can be calculated

$$
\mathbf{K}_{\mathbf{n}}=\mathbf{P}_{\mathbf{n}}^{-} \mathbf{H}_{\mathbf{n}}^{\mathbf{T}}\left[\mathbf{H}_{\mathbf{n}} \mathbf{P}_{\mathbf{n}}^{-} \mathbf{H}_{\mathbf{n}}^{\mathbf{T}}+\mathbf{R}\right]^{-\mathbf{1}}
$$

where $\mathbf{H}_{\mathbf{n}}$ is the Jacobian of the measurement function $g$. This allows the calculation of a posterior estimate of the current state

$$
\begin{aligned}
p\left(\mathbf{x}_{\mathbf{n}} \mid \mathbf{y}_{\mathbf{1}: \mathbf{n}}\right) & =\mathcal{N}\left(\hat{\mathbf{x}}_{\mathbf{n}}, \mathbf{P}_{\mathbf{n}}\right) \\
\hat{\mathbf{x}}_{\mathbf{n}} & =\hat{\mathbf{x}}_{\mathbf{n}}^{-}+\mathbf{K}_{\mathbf{n}}\left(\mathbf{y}_{\mathbf{n}}-\hat{\mathbf{y}}_{\mathbf{n}}^{-}\right) \\
\mathbf{P}_{\mathbf{n}} & =\left(\mathbf{I}-\mathbf{K}_{\mathbf{n}} \mathbf{H}_{\mathbf{n}}\right) \mathbf{P}_{\mathbf{n}}^{-} .
\end{aligned}
$$

For a QAM signal, assuming a received symbol $r_{n}=a_{n}+j b_{n}, g$ in (1) is defined by

$$
\mathbf{y}_{\mathbf{n}}=\left[\begin{array}{l}
a_{n} \cos \left(\phi_{n}+\Omega_{n} n\right)-b_{n} \sin \left(\phi_{n}+\Omega_{n} n\right) \\
a_{n} \sin \left(\phi_{n}+\Omega_{n} n\right)+b_{n} \cos \left(\phi_{n}+\Omega_{n} n\right)
\end{array}\right]+\mathbf{v}_{\mathbf{n}} .
$$

Calculating the Jacobian of (18) and requires knowledge of the current phase and received symbol. This is approximated using a hard decision on the current measurement demodulated using the prior state estimate $\left(\mathbf{y}_{\mathbf{n}} \exp \left(-j \hat{\phi}_{n}^{-}\right)\right)$.

\section{B. Particle Filter}

The particle filter estimates the PDF of the process using numerical integration; thus there are a number of particle filtering algorithms. Here, we use a generic filtering algorithm with 100 particles based on [16, Algorithm 3]. The PDF of the system state is represented as a weighted sum of $M$ particles $\mathbf{p}^{\mathrm{m}}$

$$
p(\mathbf{x}) \approx \sum_{m=1}^{M} w_{n}^{m} \delta\left(\mathbf{x}-\mathbf{p}^{\mathbf{m}}\right)
$$

where the set of weights $w^{m}$ is normalized to sum to 1 . The current state estimate $\mathbf{x}_{\mathbf{n}}$ used for phase demodulation is then calculated according to

$$
\mathbf{x}_{\mathbf{n}}=\sum_{m=1}^{M} \mathbf{p}_{\mathbf{n}}^{\mathbf{m}} w_{n}^{m} .
$$

The set of particles is drawn from an importance density $q(\mathbf{x})$ from which it must be easy to draw samples. Each weight is proportional to the fraction

$$
w^{m} \propto \frac{\pi\left(\mathbf{p}^{\mathbf{m}}\right)}{q\left(\mathbf{p}^{\mathbf{m}}\right)}
$$

where the prior density $\pi(\mathbf{x})$ must be proportional to the true PDF of the process noise $p(\mathbf{x})$ but need not be easy to draw samples from. For laser phase tracking, the initial estimate of the phase noise PDF can be sampled from quite easily and is thus used for $q(\mathbf{x})$. The initial set of particles is thus chosen from

$$
\begin{aligned}
& \mathbf{p}_{\mathbf{0}}^{\mathbf{m}} \sim q(\mathbf{x}) \\
& \mathbf{p}_{\mathbf{0}}^{\mathbf{m}} \sim \mathcal{N}\left(\mathbf{x}_{\mathbf{0}}, \mathbf{Q}\right)
\end{aligned}
$$

where $\mathbf{Q}$ is the initial guess for $\mathbf{P}$ as defined above and $\mathbf{x}_{\mathbf{0}}$ is the estimated initial system state (typically a zero vector). To incorporate information gleaned from prior measurements and known system dynamics, the set of particles is updated at each time step using the recursion equation

$$
\begin{aligned}
& \mathbf{p}_{\mathbf{n}}^{\mathbf{m}} \sim q\left(\mathbf{x}_{\mathbf{n}} \mid \mathbf{y}_{\mathbf{1}: \mathbf{n}}\right) \\
& \mathbf{p}_{\mathbf{n}}^{\mathbf{m}}=\mathbf{A p}_{\mathbf{n}-\mathbf{1}}^{\mathbf{m}}+\mathcal{N}(0, \mathbf{Q})
\end{aligned}
$$

The associated weight $w_{n}^{m}$ is updated according to

$$
\begin{aligned}
w_{n}^{m} & \propto w_{n-1}^{m} \frac{\pi\left(\mathbf{y}_{\mathbf{n}} \mid \mathbf{p}_{\mathbf{n}}^{\mathbf{m}}\right) \pi\left(\mathbf{p}_{\mathbf{n}}^{\mathbf{m}} \mid \mathbf{p}_{\mathbf{n}-1}^{\mathbf{m}}\right)}{q\left(\mathbf{p}_{\mathbf{n}}^{\mathbf{m}} \mid \mathbf{p}_{\mathbf{n}-1}^{\mathbf{m}}, \mathbf{y}_{\mathbf{n}}\right)} \\
w_{n}^{m} & \propto w_{n-1}^{m} \pi\left(\mathbf{y}_{\mathbf{n}} \mid \mathbf{p}_{\mathbf{n}}^{\mathbf{m}}\right) \\
w_{n}^{m} & \propto w_{n-1}^{m}\left(\mathbf{y}_{\mathbf{n}}-g\left(\mathbf{p}_{\mathbf{n}}^{\mathbf{m}}\right)\right)
\end{aligned}
$$

where it has been assumed that the importance density $q$ is proportional to the prior density $\pi$ (bootstrap approximation [9]). The set of particles was periodically resampled (regenerated) using systematic resampling [16, Algorithm 2] to prevent degeneracy, when all weights but one approach zero. For carrier recovery of a QAM signal, the received symbol would need to be estimated to evaluate (24); this could be done using the same strategy as in the EKF.

\section{Decision-directed Phase-locked loop}

A second-order decision-directed phase-locked loop (DDPLL) was used as a reference method to benchmark the performance of the laser rate equation based EKF in the communication experiment discussed in Section IV-B. Like the Kalman filter, the DDPLL performs both frequency offset correction and carrier phase recovery in a single stage, and thus can operate on the the same signal as the EKF. An optimized DDPLL should also perform as well as a Kalman filter for a laser with Lorentzian lineshape, which further makes it an appropriate point of reference. The primary components of a PLL are the error detector and the loop filter. The error detector used is given by [25]

$$
e_{n}=\Im\left(\hat{r}_{n} y_{n} e^{-j \phi_{n}}\right)
$$

where here $y_{n}$ is the received signal in complex form and the received symbol $\hat{r}_{n}$ is again estimated using the prior phase estimate and a hard decision. For the normalization used, this yields unity discriminator gain. The form of the loop filter was designed to be optimal for a laser with a Lorentzian lineshape. The PLL is a discrete implementation of a zero-lag proportional-integral PLL [26] with open-loop transfer function

$$
G(s)=K_{v} \frac{1+s \tau_{2}}{s^{2} \tau_{1}}
$$

where $K_{v}$ is the loop gain and $\tau_{1}$ and $\tau_{2}$ are the two loop time constants. These parameters were adjusted to yield minimum BER in the experiment. 


\section{Applications}

\section{A. Laser characterization}

Nanocavity lasers, such as photonic crystal (PhC) cavity lasers and metallic cavity lasers, have attracted interest in recent years for low-power and high-speed communication and sensing applications [27], [28]. Since these lasers tend to have low output powers, measurements of any kind suffer from low SNR. To the best of our knowledge, there has been only one report of a nanolaser linewidth measurement [29], and it was for a device with best-in-class output power. Furthermore, there have been no reports of relative intensity noise (RIN) or frequency (FM) noise power spectral density measurements, though the dynamical response has been measured in more direct ways. These more detailed spectra are necessary to understand the dynamic behavior of the laser, i.e. to determine the relaxation resonance frequency, damping constant, and linewidth enhancement factor. Coherent detection can, as a general principle, increase the sensitivity of most measurements, so some improvement relative to traditional self-homodyne/heterodyne analog techniques [30] is expected simply from using a local oscillator regardless of the digital signal processing used on the received signal. However, for the laser of interest [12], coherent detection alone was not sufficient to provide an accurate FM noise spectrum.

To ensure that the Bayesian approach to laser spectral estimation yields accurate spectra, we tested the performance of the SCL-based EKF and PF on heterodyne data from both simulated and experimentally emulated phase noise. The simulated and emulated phase noise sequences have spectral shapes similar to the FM spectrum of the PhC laser eventually tested and correspond to stable filters at the sampling rates used. The Lorentzian linewidth of the simulated spectrum is larger (around $300 \mathrm{MHz}$ ) than the Lorentzian linewidth of the emulated spectrum $(10 \mathrm{MHz})$. To emulate phase noise, we modulated an external cavity laser with a $40 \mathrm{kHz}$ Lorentzian linewidth using a $\mathrm{LiNbO}_{3}$ phase modulator and an arbitrary waveform generator at $50 \mathrm{GS} / \mathrm{s}$ [14]. Measurement noise was added using a standard (EDFA and attenuator) noise loading stage, and the signal was detected using a coherent receiver with a local oscillator with a $40 \mathrm{kHz}$ Lorentzian linewidth and a digital sampling oscilloscope at $80 \mathrm{GS} / \mathrm{s}$. Fig. 5 shows the mean squared error of the two algorithms as a function of measurement signal-to-noise ratio for simulated and emulated data. A reference method, which consists of taking the argument of the complex electric field without any digital filtering, is also shown. Simulation and experiment agree very well qualitatively. At low SNR, the measurement noise is interpreted as phase noise by the reference method, while the Bayesian filters at least partially remove its effects from the estimated phase. At high SNR, the performance of the Bayesian filtering algorithms is similar to the reference method (no DSP) because the MSE in all cases is dominated by the phase noise of the laser.

The emulated and simulated FM noise spectra were completely consistent with the rate equation model in (3), but realworld lasers are typically subject to effects that these equations do not consider (e.g. pump noise fluctuations, mechanical

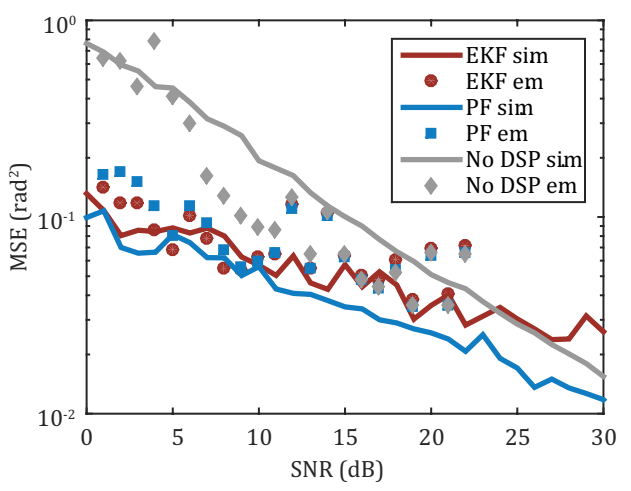

Fig. 5. Simulated and experimentally emulated MSE as a function of measurement SNR for the laser rate equation based extended Kalman filter, particle filter, and a reference method.

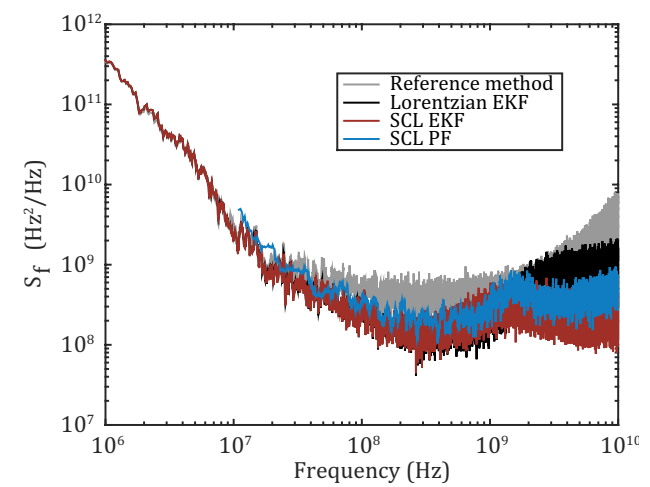

Fig. 6. Measured FM noise spectrum of a photonic crystal cavity laser with $-48 \mathrm{dBm}$ output power using a reference method and two Bayesian estimators.

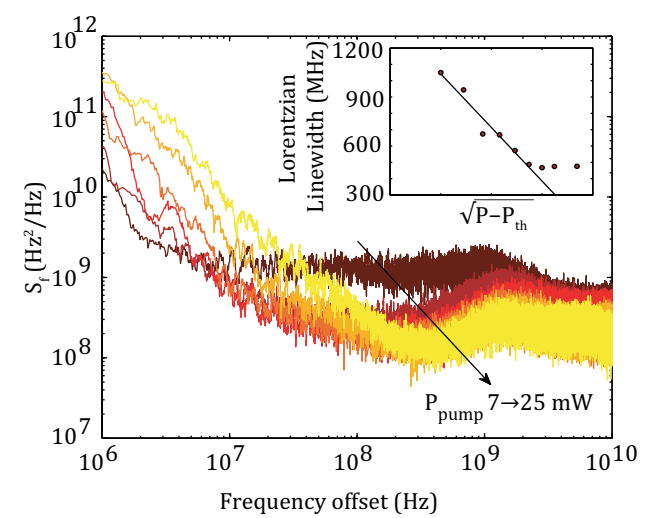

Fig. 7. Single-sided FM noise spectrum for a photonic crystal cavity laser for different values of optical pump power. Inset: Equivalent Lorentzian linewidth, calculated by averaging $\mathrm{S}_{\mathrm{f}}$ from $300 \mathrm{MHz}$ to $400 \mathrm{MHz}$, and a straight line fit.

vibrations, and temperature fluctuations). The benefit of using an adaptive algorithm like the EKF or PF in these cases, rather than a filter with fixed coefficients, is that not all model parameters need be known a priori. To demonstrate this, we used the two Bayesian filters to characterize a $\mathrm{PhC}$ laser [12]. The investigated laser was an optically pumped InP quantum dot line-defect photonic crystal cavity laser around $1550 \mathrm{~nm}$, and had a total fiber-coupled output power of 
$18 \mathrm{nW}$ [31]. The output of the $\mathrm{PhC}$ laser was amplified by an EDFA in order to reach the minimum detectable power of the coherent receiver, and the amplified spontaneous emission of the EDFA set the noise floor of the system. An initial FM noise spectrum estimate was generated using an EKF with a Lorentzian phase noise model, then used to fit coefficients for the state transition matrix for the rate equation based EKF and PF. A representative FM noise spectrum is shown in Fig. 6 for the same trace processed the Bayesian filters, the Lorentzian EKF, and the reference method. When no DSP is used, the relaxation resonance peak is not visible. When the trace is processed using the Lorentzian EKF, the relaxation resonance peak appears as a kink in the spectrum; the only real conclusions that can be drawn from this spectrum are that the damping factor is large and that the resonance frequency is around $3 \mathrm{GHz}$. This is sufficiently accurate to specify a state transition matrix that allows the SCL-based EKF and PF to perform better than the Lorentzian EKF: in the traces processed using the SCL-based Bayesian filters, the relaxation resonance peak is clearly visible. Fig. 7 shows the measured FM noise spectrum of the laser as the pump power is increased from near threshold $\left(\mathrm{P}_{\mathrm{th}}\right)$ to $4 \mathrm{P}_{\mathrm{th}}$. As expected, the linewidth $\Delta \nu$ (shown as an inset) decreases with increasing pump power, and the relaxation resonance peak moves to higher frequencies. These two general trends are expected and provide further evidence that the DSP is providing an increase in sensitivity rather than simply imposing a shape on the noise spectrum. This indicates that this is a particularly powerful method for systematically characterizing lasers with low output power whose behavior is consistent with a rate equation model.

\section{B. Communication}

Rising datacenter traffic is driving metro-area links to operate at higher and higher data rates [32]. Coherent modulation formats with high spectral efficiency are a proven approach for providing high bitrates with moderate-bandwidth electronic and photonic components, but the coherent transceivers currently used for long-haul applications are too large for use in metro area and datacenter networks. A coherent transceiver for Ethernet would most likely have to use an integrated semiconductor tunable laser rather than a relatively bulky external cavity laser (ECL). Because of this, there has been great progress in recent years toward compact integrated devices with narrow linewidths [33], [34]. However, the distinctive FM noise spectrum of an integrated SCL can cause standard carrier phase recovery algorithms to fail, even when the $3 \mathrm{~dB}$ linewidth of the laser is theoretically narrow enough to allow penalty-free transmission [14]. When the laser lineshape is far from Lorentzian, the EKF formulation discussed in Section II can yield a dramatically superior performance compared to CPR algorithms based on Lorentzian models.

We tested filter performance for a variety of damping frequencies experimentally and in simulation [13], [14]. Phase noise sequences were emulated experimentally using the technique described in Section IV-A and [14], [35]. A 28 GBd electrical multilevel signal for the transmitter was generated by actively combining two de-correlated $2^{15}-1$ pseudo-random bit sequences, and drove a $25 \mathrm{GHz}$ I-Q modulator to obtain a single-polarization 16 QAM signal. After polarization multiplexing emulation, this was transmitted over a short length of fiber through a standard EDFA and attenuator noise-loading stage to a coherent receiver with $42 \mathrm{GHz}$ analog bandwidth and a sampling rate of $80 \mathrm{GHz}$. The local oscillator, an external cavity laser with $40 \mathrm{kHz}$ linewidth, was frequencylocked to the incoming signal, which resulted in negligible FM noise from DC to about $30 \mathrm{MHz}$ but the desired phase noise at all higher frequencies. For both the simulation and the experiment, receiver-side DSP [8] consisted of IQ imbalance compensation, retiming, equalization at two samples per symbol using the multi-modulus algorithm, and finally the carrier recovery algorithm of interest. The effect of cycle slips was removed from simulated and experimental results by discarding blocks of data containing burst errors.

Fig. 8 shows the experimental BER performance of polarization multiplexed $28 \mathrm{GBd} 16 \mathrm{QAM}$ for an SCL with a Lorentzian linewidth of $500 \mathrm{kHz}, 1 \mathrm{GHz}$ relaxation resonance frequency, and $0.1 \mathrm{~ns}$ damping factor when demodulated with the decision-directed phase-locked loop, the laser rate equation based Kalman filter, and an EKF that uses a Lorentzian laser model [10]. The DDPLL feedback parameters were optimized to yield the lowest BER: $K_{v}=11 \mathrm{e} 9, \tau_{1}=\tau_{2}=16 \mathrm{~ns}$. This resulted in a loop bandwidth around $100 \mathrm{MHz}$ and accordingly, very little suppression of the phase noise around the relaxation resonance peak at $1 \mathrm{GHz}$. A stable SCL Kalman filter cannot be formulated for a laser with these parameters, so $K$ was set to $1.4 \mathrm{~ns}$. The SCL Kalman filter performs close to the zeroadded phase noise case, while the other two carrier recovery techniques do not provide BERs below the FEC limit even at $30 \mathrm{~dB}$ OSNR. The FM noise spectra recovered phase from the SCL EKF and DDPLL are shown in Fig. 9 (a) along with the transmitter FM noise spectrum. The SCL EKF accurately tracks the phase in the vicinity of the relaxation resonance peak, while the DDPLL under-estimates the fluctuation in that part of the noise spectrum by an order of magnitude. The associated constellations at high OSNR are shown in Fig. 9 (b) and confirm that the large penalty for using the DDPLL is primarily due to phase noise. This behavior is predicted in Fig. 3, which shows that the best stable filter for a laser with this FM spectrum will yield lower MSE than a filter based on a Lorentzian model. In the experiment, it was assumed

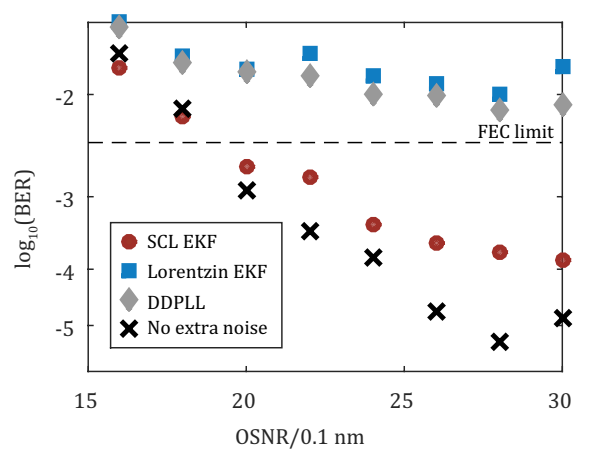

Fig. 8. BER as a function of OSNR for different carrier recovery algorithms. 


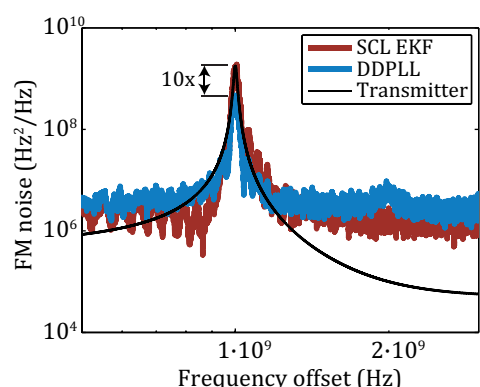

(a)
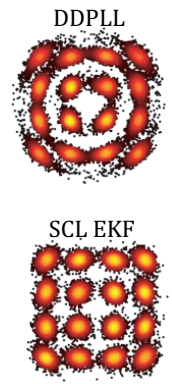

(b)
Fig. 9. (a) FM spectrum of recovered phase for transmitter and for different carrier recovery algorithms. (b) Associated constellations (top: DDPLL, bottom: laser Kalman)

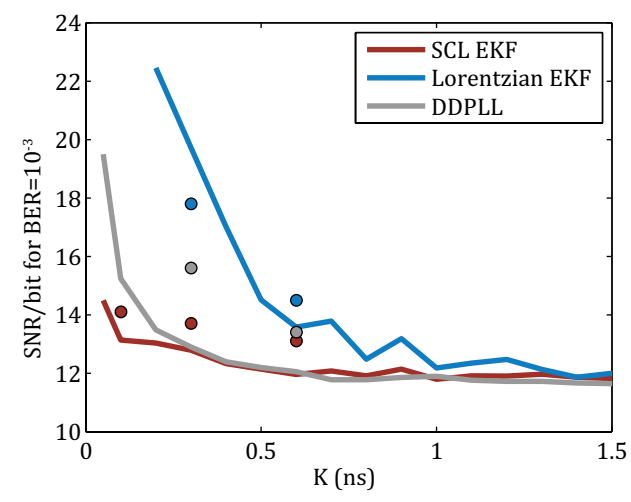

Fig. 10. SNR requirements for the three carrier recovery techniques as a function of damping factor $\left(f_{R}=1 \mathrm{GHz}\right.$ and $\left.\Delta \nu=500 \mathrm{kHz}\right)$

the relaxation resonance frequency was known at the receiver, though it can also be estimated using a generic filter and the bootstrapping method discussed in Section IV-A; even when the DDPLL does not produce a BER below the FEC limit, the relaxation resonance peak appears in the spectrum of the demodulated phase.

Fig. 10 shows the measured and calculated signal to noise ratio (per bit) required to reach a BER of $1 \mathrm{e}-3$ for all three carrier recovery techniques as a function of damping factor. The relaxation resonance frequency and Lorentzian linewidth were again $1 \mathrm{GHz}$ and $500 \mathrm{kHz}$, respectively. As the damping factor is increased, the optimum laser model approaches the stable filtering region, which yields about $1 \mathrm{~dB}$ improvement in SCL Kalman performance as the damping factor is increased from $0.1 \mathrm{~ns}$ to $0.6 \mathrm{~ns}$. For the lowest damping factors, the DDPLL and Lorentzian Kalman are substantially worse than the SCL Kalman, for the reason discussed above. It is worth noting that the Lorentzian Kalman and DDPLL do not behave exactly the same way even though there is very little difference between the two algorithms. This is because the DDPLL feedback gain was optimized, while the Lorentzian Kalman adjusts the gain based on the learned phase error variance of the incoming signal. When the damping factor is very small, the variance that the Lorentzian Kalman filter estimates tends to be much larger than the correct value (this is expected [1]), and thus picks excessively large values for the feedback gain. For high damping factors, there is little difference between the three techniques. This is because the area where a Lorentzian model is competitive with the SCL model nearly covers the full $K-f_{R}$ plane. The experimental data follows the same qualitative trend as the simulation. The penalty for the DDPLL at low $K$ is substantially larger in the experiment as compared to the simulation: it is greater than $8 \mathrm{~dB}$ for $K=0.1 \mathrm{~ns}$ in the experiment, but only $2 \mathrm{~dB}$ in the simulation. For resonance frequencies $3 \mathrm{GHz}$ and higher, the difference in performance between the SCL Kalman and the Lorentzian model based methods is less pronounced [14]. This is because the difference in $V$ in (12) for the best stable SCL model and the Lorentzian is much smaller.

\section{CONCLUSION}

We developed a novel carrier phase recovery technique based on a rate equation model and Bayesian filtering for digital coherent systems that use semiconductor lasers. It was shown that this technique is most useful in two scenarios: first, when filter stability is not a concern but measurement signalto-noise ratio is very low, and second, when the relaxation resonance frequency and damping factor are both low. This first scenario occurs in measuring the FM noise spectrum of lasers with low output power, and it was demonstrated that the technique can work even when an accurate laser model is not initially available. The second scenario can occur in coherent communication systems employing semiconductor lasers at the transmitter, and we showed that the largest system gain is available in operating regimes where traditional carrier recovery techniques are most likely to fail.

\section{ACKNOWLEDGMENT}

The authors thank Kresten Yvind, Louisa Ottaviano, Elizaveta Semenova, and Yi Yu for device fabrication.

\section{REFERENCES}

[1] K. Kikuchi and T. Okoshi, "Measurement of FM noise, AM noise, and field spectra of $1.3 \mu \mathrm{m}$ InGaAsP DFB lasers and determination of the linewidth enhancement factor," IEEE J. Quantum Electron., vol. QE-21, no. 11 , pp. 1814-1818, 1985.

[2] C. Henry, "Phase noise in semiconductor lasers," J. Lightw. Technol., vol. 4, no. 3, pp. 298-311, Mar. 1986.

[3] E. Ip and J. M. Kahn, "Carrier synchronization for 3- and 4-bit-persymbol optical transmission," J. Lightw. Technol., vol. 23, no. 12, pp. 4110-4124, Dec. 2005.

[4] M. G. Taylor, "Phase estimation methods for optical coherent detection using digital signal processing," J. Lightw. Technol., vol. 27, no. 7, pp. 901-914, Apr. 2009.

[5] K. Kikuchi, "Characterization of semiconductor-laser phase noise and estimation of bit-error rate performance with low-speed offline digital coherent receivers," Opt. Express, vol. 20, no. 5, pp. 5291-302, Feb. 2012.

[6] T. N. Huynh, L. Nguyen, and L. P. Barry, "Phase noise characterization of SGDBR lasers using phase modulation detection method with delayed self-heterodyne measurements," J. Lightw. Technol., vol. 31, no. 8, pp. 1300-1308, Apr. 2013.

[7] T. Okamoto and F. Ito, "Laser phase noise characterization using parallel linear optical sampling," J. Lightw. Technol., vol. 32, no. 18, pp. 31193125, Sep. 2014.

[8] S. J. Savory, "Digital coherent optical receivers: Algorithms and subsystems," IEEE J. Quantum Electron., vol. 16, no. 5, pp. 1164-1179, Sep. 2010.

[9] A. J. Haug, "A tutorial on Bayesian estimation and tracking techniques applicable to nonlinear and non-Gaussian processes," Mitre, Tech. Rep., 2005. 
[10] D. Zibar, L. Carvalho, M. Piels, A. Doberstein, J. Diniz, B. Nebendahl, C. Franciscangelis, J. Estaran, H. Haisch, N. G. Gonzalez, J. de Oliveira and I. Tafur Monroy, "Application of machine learning techniques for amplitude and phase noise characterization," J. Lightw. Technol., vol. 33, no. 7, pp. 1333-1343, Apr. 2015.

[11] M. Piels, I. Tafur Monroy, and D. Zibar, "Laser characterization with advanced digital signal processing," in Proc. SPIE, 2015, pp. 9388-8.

[12] M. Piels, W. Xue, C. Schäffer, Y. Yu, E. Semenova, L. Ottaviano, K. Yvind, I. Tafur Monroy, and D. Zibar, "Highly sensitive photonic crystal cavity laser noise measurements using Bayesian filtering," in OFC/NFOEC Techn. Digest. Opt. Fiber Commun. Conf., 2015., p. W2A.28.

[13] M. Piels, M. I. Olmedo, X. Pang, R. Schatz, G. Jacobsen, S. Popov, and D. Zibar, "Rate-equation-based phase recovery for semiconductor laser coherent transmitters," in OFC/NFOEC Tech. Digest. Opt. Fiber Commun. Conf., 2015., p. W1E.7.

[14] M. I. Olmedo, X. Pang, M. Piels, R. Schatz, G. Jacobsen, S. Popov, I. Tafur Monroy, and D. Zibar, "Carrier recovery techniques for semiconductor laser frequency noise for 28 GBd DP-16QAM," in OFC/NFOEC Tech. Digest. Opt. Fiber Commun. Conf., 2015., p. Th2A.10.

[15] W.-T. Lin and D.-C. Chang, "Adaptive carrier synchronization using decision-aided Kalman filtering algorithms," IEEE Trans. Consum. Electron., vol. 53, no. 4, pp. 1260-1267, Nov. 2007.

[16] M. S. Arulampalam, S. Maskell, N. Gordon, and T. Clapp, "A tutorial on particle filters for online nonlinear/non-Gaussian Bayesian tracking," IEEE Trans. Signal Process., vol. 50, no. 2, pp. 174-188, 2002.

[17] E. A. Wan and R. van der Merwe, "The unscented Kalman filter for nonlinear estimation," in Adaptive Syst. for Signal Process., Commun., and Control Symp., Lake Louise, Alberta, Canada, 2000, pp. 153 - 158.

[18] E. Panayırcı, H. A. Çırpan, M. Moeneclaey, and N. Noels, "Blind phase noise estimation and data detection based on SMC technique and unscented filtering," in Eur. Signal Process. Conf. (EUSIPCO-2006), Florence, Italy.

[19] D. J. Higham, "An algorithmic introduction to numerical simulation of stochastic differential equations," SIAM Review, vol. 43, no. 3, pp. 525546, Jan. 2001.

[20] K. Kikuchi, "Effect of 1/f-type FM noise on semiconductor-laser linewidth residual in high-power limit," IEEE J. Quantum Electron., vol. 25, no. 4, pp. 684-688, Apr. 1989.

[21] W. Shieh and K.-P. Ho, "Equalization-enhanced phase noise for coherent-detection systems using electronic digital signal processing," Opt. Express, vol. 16, no. 20, p. 15718, Sep. 2008.
[22] L. M. Pessoa, H. M. Salgado, and I. Darwazeh, "Performance evaluation of phase estimation algorithms in equalized coherent optical systems," IEEE Photon. Technol. Lett., vol. 21, no. 17, pp. 1181-1183, Sep. 2009.

[23] F. van der Heijden, R. Duin, D. de Ridder, and D. M. J. Tax, Classification, Parameter Estimation and State Estimation. Chichester, UK: John Wiley and Sons, Ltd., 2004.

[24] L. Ljung, System Identification: Theory for the User. Englewood Cliffs, NJ: Prentice-Hall, Inc., 1987.

[25] H. Meyr, M. Moeneclaey, and S. Fechtel, Digital Communication Receivers: Synchronization, Channel Estimation, and Signal Processing. John Wiley \& Sons, Inc., 1999, vol. 2.

[26] R. Best, Phase-Locked Loops: Design, Simulation, and Applications, 3rd ed. McGraw-Hill, 1997.

[27] M. T. Hill, "Status and prospects for metallic and plasmonic nanolasers," J. Opt. Soc. Am. B, vol. 27, no. 11, p. B36, Oct. 2010.

[28] S. Matsuo, T. Sato, K. Takeda, A. Shinya, K. Nozaki, H. Taniyama, M. Notomi, K. Hasebe, and T. Kakitsuka, "Ultralow operating energy electrically driven photonic crystal lasers," IEEE J. Quantum Electron., vol. 19, no. 4, pp. 4900311-4900311, Aug. 2013.

[29] J. Kim, A. Shinya, K. Nozaki, H. Taniyama, C.-H. Chen, T. Sato, S. Matsuo, and M. Notomi, "Narrow linewidth operation of buriedheterostructure photonic crystal nanolaser," Opt. Express, vol. 20, no. 11, pp. 11 643-51, May 2012.

[30] D. Derickson, Fiber optic test and measurement. Upper Saddle River, New Jersey: Prentice Hall, Inc., 1998.

[31] W. Xue, in preparation.

[32] "Cisco global cloud index: Forecast and methodology, 2012-2017," 2013, White Paper.

[33] J. C. Hulme, J. K. Doylend, and J. E. Bowers, "Widely tunable Vernier ring laser on hybrid silicon," Opt. Express, vol. 21, no. 17, pp. 19718 22, Aug. 2013.

[34] K. Sato, N. Kobayashi, M. Namiwaka, K. Yamamoto, T. Kita, H. Yamada, and H. Yamazaki, "High output power and narrow linewidth silicon photonic hybrid ring-filter external cavity wavelength tunable lasers," in 2014 The Eur. Conf. on Opt. Commun. (ECOC), Sep., pp. $1-3$.

[35] M. I. Olmedo, X. Pang, A. Udalcovs, R. Schatz, D. Zibar, G. Jacobsen, S. Popov, I. Tafur Monroy, and D. Zibar, "Impact of carrier induced frequency noise from the transmitter laser on 28 and 56 Gbaud DPQPSK metro links," in Asia Commun. and Photonics Conf. 2014, p. ATh1E.1. 